\title{
Optimal beam size for light delivery to absorption-enhanced tumors buried in biological tissues and effect of multiple-beam delivery: a Monte Carlo study
}

\author{
Lihong V. Wang, Robert E. Nordquist, and Wei R. Chen
}

\begin{abstract}
Optimal laser light delivery into turbid biological tissues was studied by using Monte Carlo simulations based on the delta-scattering technique. The goal was to deliver efficiently the maximum amount of optical power into buried tumors being treated while avoiding potential damage to normal tissue caused by strong optical power deposition underneath the tissue surface illuminated by the laser beam. The buried tumors were considered to have much higher absorption than the surrounding normal tissue because of selective uptake of the absorption-enhancement dye. The power delivering efficiency to buried tumors was investigated for various diameters of the laser beam. An optimal beam diameter was estimated to achieve the maximum product of the power coupling efficiency and the power delivered to the buried tumor. The distribution of power deposition was simulated for single-beam delivery and multiple-beam delivery as well. The simulated results showed that with an appropriate dye enhancement and an optimal laser delivery configuration, a high selectivity for laser treatment of tumor could be achieved. (c) 1997 Optical Society of America

Key words: Monte Carlo, light delivery, optical therapy, turbid media, biological tissues.
\end{abstract}

\section{Introduction}

Laser-tissue interactions and their therapeutic applications is a fast-growing research area. Biological tissues are turbid media in which light attenuates rapidly because of light absorption augmented by strong light scattering. As a consequence, deeply buried tumors usually receive much less optical power than the subsurface normal tissues, which hampers efficacious optical treatment of tumors. The tumor treatment can be improved by maximizing the power delivered to the target tumors while avoiding damaging subsurface normal tissues. Specifi-

L. V. Wang (corresponding author) is with the Optical Imaging Laboratory, Bioengineering Program, Texas A\&M University, College Station, Texas 77843-3120. W. R. Chen is with the Oklahoma School of Science and Mathematics, 1141 North Lincoln Boulevard, Oklahoma City, Oklahoma 73104 and the Department of Physics and Astronomy, University of Oklahoma, Norman, Oklahoma 73109. R. E. Nordquist is with Wound Healing of Oklahoma, Inc., 3939 N. Walnut Street, Oklahoma City, Oklahoma 73105.

Received 6 March 1997; revised manuscript received 13 June 1997.

0003-6935/97/318286-06\$10.00/0

(C) 1997 Optical Society of America cally, several steps may be taken. First, the absorption coefficient of tumors may be significantly increased by infusing dyes into the tumors. Second, the wavelength of the laser light may be selected to maximize the ratio between the absorption of the tumor and that of the surrounding normal tissue. Third, the light delivery scheme may be optimized to maximize the power absorption by the target tumors. We concentrate on the third approach in this paper.

In previous studies the effect of the diameter of the laser beam was investigated while the power density of the laser beam was kept constant.1,2 In this paper, Monte Carlo simulations were used to identify the optimal diameters of the laser beam and to investigate the effect of multiple-beam delivery compared with single-beam delivery in the condition that the location of the buried tumor is known and the absorption of the tumor is enhanced.

\section{Method}

Monte Carlo simulations of light transport in tissues have been implemented previously for simple tissue geometry. ${ }^{3-8}$ To compute light distributions according to tissue geometry and optical properties, including refractive index $n$, absorption coefficient $\mu_{a}$, scattering coefficient $\mu_{s}$, and anisotropy factor $g$, we 
have written a Monte Carlo program in $\mathrm{C}$ for tissues with buried objects. We used the delta-scattering technique ${ }^{9}$ for photon tracing to greatly simplify the algorithm because this technique allows a photon packet to be traced without directly dealing with photon crossings of interfaces between different types of tissues. This technique can be used only for refractive-index-matched tissues, although it allows the ambient clear media (e.g., air) and the tissue to have mismatched refractive indices. Since most soft tissues have similar indices of refraction, this limitation does not pose a problem in our simulation.

We assume that the tissue system has multiple tissue types with identical refractive indices. The interaction coefficient of the $i$ th tissue type, defined as the sum of $\mu_{a}$ and $\mu_{s}$, is denoted by $\mu_{i}$. The deltascattering technique applied to light transport in biological tissues is briefly summarized:

(1) Define a majorant interaction coefficient $\mu_{m}$, where $\mu_{m} \geq \mu_{i}$ for all $i$. In this study, $\mu_{m}$ was set to the maximum $\mu_{i}$ among all tissue types.

(2) Select a step size $R$ between two consecutive interactions based on the majorant interaction coefficient,

$$
R=-\ln (\xi) / \mu_{m},
$$

where $\xi$ is a uniformly distributed random number between 0 and $1(0<\xi \leq 1)$. Then, determine the tentative next collision site $\mathbf{r}_{k}{ }^{\prime}$ by

$$
\mathbf{r}_{k}^{\prime}=\mathbf{r}_{k-1}+R \mathbf{u}_{k-1}
$$

where $\mathbf{r}_{k-1}$ is the current site and $\mathbf{u}_{k-1}$ is the direction of the flight. The direction of the flight is determined according to the probability distributions of deflection and azimuthal angles at each interaction site. $^{7}$

(3) Play a rejection game:

(a) Get another random number $\eta$, which is uniformly distributed between 0 and $1(0<\eta \leq$ 1).

(b) If $\eta \leq \mu_{i}\left(\mathbf{r}_{k}{ }^{\prime}\right) / \mu_{m}$, i.e., with a probability of $\mu_{i}\left(\mathbf{r}_{k}{ }^{\prime}\right) / \mu_{m}$, accept this point as a real interaction site $\left(\mathbf{r}_{k}=\mathbf{r}_{k}{ }^{\prime}\right)$.

(c) Otherwise, do not accept $\mathbf{r}_{k}^{\prime}$ as a real interaction site but select a new path starting from $\mathbf{r}_{k}{ }^{\prime}$ with the unchanged direction $\mathbf{u}_{k-1}$ [i.e., set $\mathbf{r}_{k-1}=\mathbf{r}_{k}{ }^{\prime}$ and return to step (2)].

At each real interaction site, a fraction $\mu_{a} /\left(\mu_{a}+\mu_{s}\right)$ of the photon packet is absorbed and the rest are scattered. The tracing continues until either the weight of the photon packet is below a set threshold or the packet exits the tissue system. A subthreshold photon packet experiences a Russian roulette to conserve energy. Since step (3) is very efficient, the delta-scattering technique is comparable with the standard Monte Carlo simulation methods in computational speed. The detailed treatment of photon tracing after step (3) is similar to that in Ref. 7 and is not repeated here.

The validity of step (3) can be easily understood when an imaginary interaction event is introduced that changes neither the weight nor the direction of the photon. This definition implies that such imaginary interactions are not physically observable; i.e., they can be introduced with any interaction coefficient at any point. We may assume that the majorant interaction coefficient $\mu_{m}$ is a sum of the real $\mu_{R e}$ and imaginary $\mu_{\mathrm{Im}}$ interaction coefficients, where the real interaction coefficient $\mu_{\mathrm{Re}}$ is $\mu_{i}\left(\mathbf{r}_{k}{ }^{\prime}\right)$. In the procedure outlined above, a fraction of the interactions,

$$
1-\mu_{\mathrm{Re}} / \mu_{m}=\mu_{\mathrm{Im}} / \mu_{m}
$$

are imaginary interactions. From another point of view, it is easy to see that on the average, for every $\mu_{m}$ total interactions, there will be $\mu_{\mathrm{Re}}$ interactions accepted as real interactions. The mean free path for the majorant interactions in the delta-scattering method is $1 / \mu_{m}$, and the mean free path for the real interactions in the direct method is $1 / \mu_{\mathrm{Re}}$. Therefore the photon moves to the correct interaction site when one uses the delta-scattering technique as it would when one uses the direct method because

$$
\mu_{m}\left(1 / \mu_{m}\right)=\mu_{\mathrm{Re}}\left(1 / \mu_{\mathrm{Re}}\right),
$$

where the left-hand side means the average distance traveled by the photon packet with $\mu_{m}$ total steps or $\mu_{\mathrm{Re}}$ real interactions in the delta-scattering method, and the right-hand side means the average distance traveled with $\mu_{R e}$ real interactions in the direct method.

During the tracing of each weighted photon, ${ }^{7}$ the light absorption, reflection, or transmission was correspondingly scored into different arrays according to the spatial positions of the photon. Multiple photons are traced to achieve an acceptable statistical variation. For this study, 100,000 photons were traced.

This Monte Carlo program was used to simulate the power deposition for tissue configurations as shown in Fig. 1. Figure 1(a) shows a single-beam delivery scheme to a tissue slab with a tumor buried in the middle between the top and bottom tissue surfaces. The lateral dimensions in the $x y$ plane were considered optically infinite, i.e., much greater than the penetration depth of light. Figure 1(b) illustrates a multiple-beam delivery scheme to a tissue cube with a tumor buried in the center. One or more of the four beams may be selected to illuminate the tissue while the other beams are blocked.

\section{Results}

The single-beam delivery scheme as shown in Fig. 1(a) was studied with the Monte Carlo program. The total power absorption by the buried tumor $P_{\mathrm{tu}^{-}}$ mor in watts and the maximum deposited power density in the tissue $Q_{\max }$ in $\mathrm{W} / \mathrm{cm}^{3}$ were simulated for different radii of the laser beam while the total incident power $P_{s}$ was kept constant (Fig. 2). 

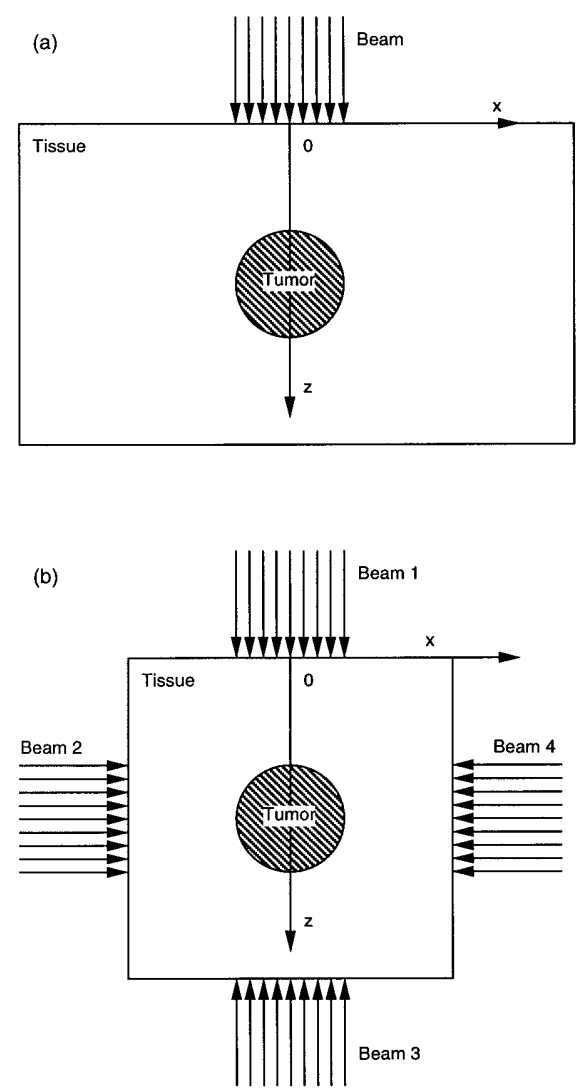

Fig. 1. Configurations of the biological tissue and laser beams: (a) single-beam delivery to a wide tissue slab; (b) multiple-beam delivery to a tissue cube. The tumor was a sphere centered in the background tissue and aligned with the center of the laser beam. In both cases, the optical properties of the background tissue were: absorption coefficient, $\mu_{a}=0.1 \mathrm{~cm}^{-1}$; scattering coefficient, $\mu_{s}=$ $100 \mathrm{~cm}^{-1}$; anisotropy, $g=0.9$. The optical properties of the tumor were: $\mu_{a}=1 \mathrm{~cm}^{-1}, \mu_{s}=100 \mathrm{~cm}^{-1}$, and $g=0.9$. The diameter of the tumor was $1 \mathrm{~cm}$. The thickness of (a) the tissue slab and (b) the side of the tissue cube were both $3 \mathrm{~cm}$.

In determining the maximum power density deposited in the tissue immediately under the laser beam, the total incident power $P_{s}$ was set to $1 \mathrm{~W}$ for convenience. After the data point at zero radius of beam was removed, the following relationship was fitted into the Monte Carlo simulated $Q_{\max }$ data by using the least-squares fit:

$$
Q_{\max }=0.156 r^{-1.70},
$$

where $r$ was the radius of the beam in centimeters, and $Q_{\max }$ was in $\mathrm{W} / \mathrm{cm}^{3}$. Since the incident power $P_{s}$ was set to $1 \mathrm{~W}$ in this simulation, $P_{\text {tumor }}$ was equal to the fraction of the incident power that was absorbed by the tumor $\alpha_{\text {tumor }}$. In general, $\alpha_{\text {tumor }}$ was the ratio between $P_{\text {tumor }}$ and $P_{s}$, i.e., the efficiency of power coupling from the incident laser beam into the tumor.

The power absorption by the buried tumor $P_{\text {tumor }}$ was also simulated for different radii of the laser beam while the total incident power $P_{s}$ was varied to keep the maximum power deposition $Q_{\max }$ constant

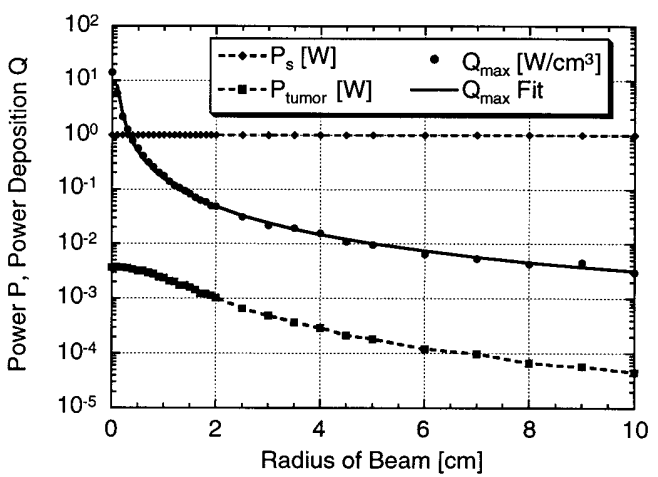

Fig. 2. Power absorption $P_{\text {tumor }}$ (squares) by the tumor and maximum power deposition $Q_{\max }$ (circles) as a function of the radius of the laser beam in single-beam delivery to a tissue slab while the power of the incident laser beam $P_{s}$ (diamonds) was set to $1 \mathrm{~W}$. The fit to $Q_{\max }$ by Eq. (5) is shown by the solid curve. Refer to Fig. 1 (a) for the optical and geometric parameters.

(Fig. 3). For convenience, $Q_{\max }$ was set to $1 \mathrm{~W} / \mathrm{cm}^{3}$. The product of $\alpha_{\text {tumor }}$ and $P_{\text {tumor }}$ was also shown in Fig. 3. There existed an optimal radius of the laser beam to maximize this product, where the optimal radius was between 1 and $1.5 \mathrm{~cm}$ for this tissue configuration. The following relationship was fitted into the Monte Carlo simulated $P_{s}$ data for $r \geq 4 \mathrm{~cm}$ by using the least-squares fit:

$$
P_{s}=34.0+2.86 r^{2} .
$$

An analytical estimate of the optimal radius may be obtained by using the geometry in Fig. 4 . The dashed horizontal line underneath the tissue surface was one transport mean free path $L_{t}{ }^{\prime}$ below the surface. To the first-order approximation, the laser beam may be modeled as isotropic point sources one transport mean free path below the surface. ${ }^{10-12}$

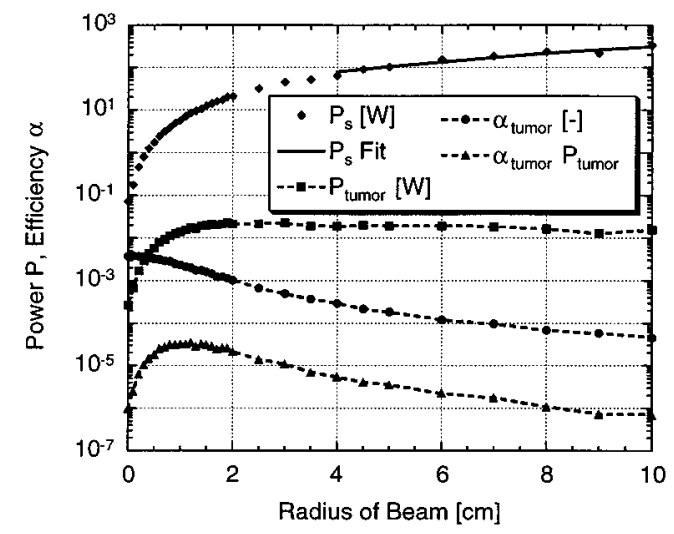

Fig. 3. Diamonds, power of the incident laser beam $P_{s}$; solid curve, fit to $P_{s}$ for a radius greater than $4 \mathrm{~cm}$. Squares, power absorption by the tumor $P_{\text {tumor }}$. Circles, power coupling efficiency $\alpha_{\max }$. Triangles, product of the power coupling efficiency and power absorption by the tumor $\alpha_{\text {tumor }} P_{\text {tumor }}$ as a function of the radius of the laser beam in the single-beam delivery to a tissue slab while the maximum power deposition $Q_{\max }$ was kept constant at 1 $\mathrm{W} / \mathrm{cm}^{3}$. Refer to Fig. 1(a) for the optical and geometric parameters. 


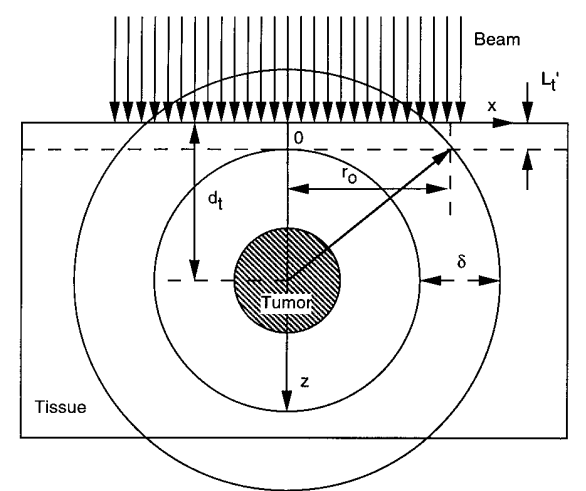

Fig. 4. Schematic of single-beam delivery for the derivation of the estimated optimal radius of the laser beam.

The coupling efficiency of power from these equivalent point sources to the tumor varied with the horizontal positions of the point sources. The coupling efficiency was the greatest at the center $(x=0)$ and decreased as $x$ increased. When the distance between the point source and the center of the tumor was increased by one penetration depth $\delta$ compared with the shortest distance at $x=0$, the contribution of the point source would be significantly reduced. The horizontal coordinate corresponding to this distance was used to estimate the optimal radius of the laser beam $r_{o}$. Two circles were drawn in Fig. 4 around the center of the tumor for the derivation, where the radii of the small and large circles were $d_{t}$ $-L_{t}{ }^{\prime}$ and $d_{t}-L_{t}{ }^{\prime}+\delta$, respectively. The optimal radius was derived as

$$
r_{o}=\left[\delta\left(2 d_{t}-2 L_{t}^{\prime}+\delta\right)\right]^{1 / 2},
$$

where $d_{t}$ is the distance between the center of the tumor and the upper tissue surface and $\delta$ is the penetration depth.

The multiple-beam delivery scheme as shown in Fig. 1(b) was studied with this Monte Carlo program. The distributions of power deposition are shown in Figs. 5(a), 5(b), and 5(c) for beam radii of $0,0.5$, and $1 \mathrm{~cm}$, respectively. The power of each beam was set to $1 \mathrm{~W}$ for convenience. The plots described the $\log _{10}$ (absorption), where the absorption was the power deposition in units of $\mathrm{W} / \mathrm{cm}^{3}$. The general trend was that the more beams turned on for the light delivery, the more homogeneous and intense the power deposition in the tumor. The broader the beams, the more homogeneous the power deposition in the tumor while it would be most effective if the optimal radius as described by Eq. (7) was chosen.

\section{Conclusions and Discussion}

Figure 2 shows that the maximum power deposition $Q_{\max }$ decreased with increasing beam size while the power of the incident laser beam $P_{s}$ was kept constant. As the beam broadened, the edge of the beam contributed less and less to the maximum power deposition $Q_{\max }$ near the center of the beam. The relationship between the maximum power deposition and
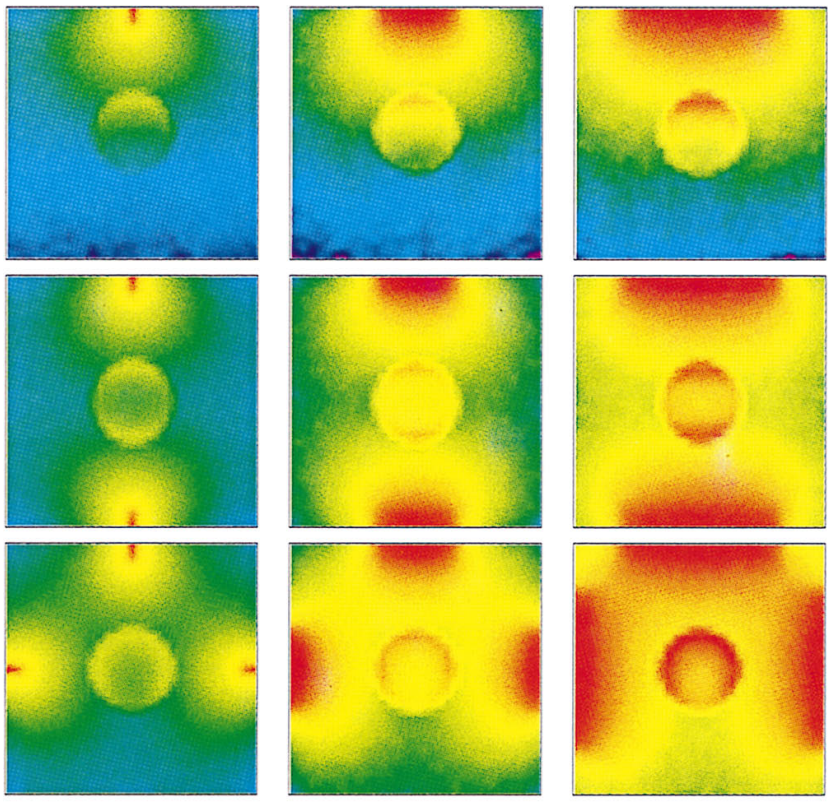

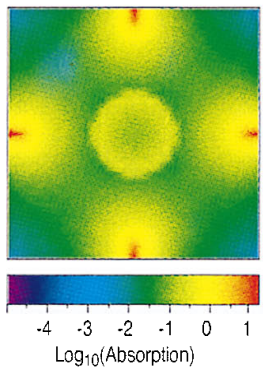

(a)

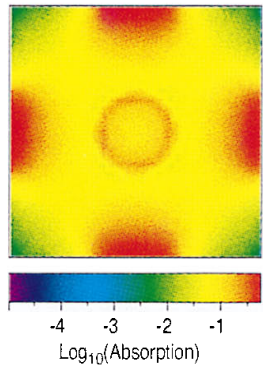

(b)

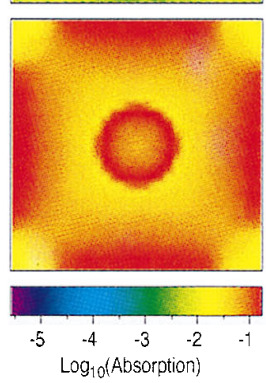

(c)
Fig. 5. False-color plots of the deposited power density distributions in $\mathrm{W} / \mathrm{cm}^{3}$ in the tissue cube in log scale. The radii of the beam were (a) $0 \mathrm{~cm}$, (b) $0.5 \mathrm{~cm}$, and (c) $1 \mathrm{~cm}$. Each column plots for one through four-beam delivery. The power of each incident laser beam $P_{s}$ was set to $1 \mathrm{~W}$. Refer to Fig. 1(b) for the optical and geometric parameters.

the radius of the beam was described by the empirical expression in Eq. (5). Since the power density $\phi$ of the laser beam was inversely proportional to the area of the beam while the power of the beam was kept constant, Eq. (5) may be reformulated as

$$
Q_{\max }=0.156 r^{-1.70}=0.156\left(r^{-2}\right)^{0.85} \propto \phi^{0.85} .
$$

Therefore the maximum power deposition $Q_{\max }$ was not proportional to the power density $\phi$ as one might have anticipated but proportional to the power density raised to a power of less than unity while the power of the incident laser beam $P_{s}$ was held constant.

Although Fig. 2 was generated for a constant incident power, $P_{s}=1 \mathrm{~W}$, the results in the figure may be scaled according to the actual incident power $P_{s}$. Based on the actual power $P_{s}$ of the laser beam, an appropriate radius of beam may be selected to ensure that the maximum power deposition $Q_{\max }$ is below the damage threshold $Q_{\mathrm{th}}$.

Figure 3 shows that when the maximum power deposition $Q_{\max }$ was kept constant, the power of the 
incident laser beam $P_{s}$ increased with the radius of the beam rapidly at small beam radii but became linear with the square of the beam radius. Since the power coupling efficiency from the edge of the beam to the site of the maximum power deposition $Q_{\max }$ near the center of the beam decreased as the beam broadened, one may think generally that only the inner portion of a wide beam contributed to the maximum power deposition $Q_{\max }$ and the outer portion made a negligible contribution. The power of the outer portion was proportional to the area of this portion and the power density of the beam. The power density of a sufficiently wide beam would be approximately constant with an increasing beam radius while the maximum power deposition $Q_{\max }$ was held constant. Therefore the incident power $P_{s}$ of a wide beam was expected to increase with the square of the radius or with the area of the beam.

Figure 3 also showed that the power deposited into the tumor $P_{\text {tumor }}$ increased rapidly with increasing beam size but gradually reached a plateau for a beam radius greater than $1 \mathrm{~cm}$. The reason was that the efficiency of power transfer $\alpha_{\text {tumor }}$ from the incident laser beam to the tumor decreased with the radius of the beam. The product of $\alpha_{\max }$ and $P_{\text {tumor }}$ reached a maximum near 1-1.5-cm radius, which encompassed an optimal radius.

The optimal radius may be estimated with Eq. (7) for the Monte Carlo simulations in Fig. 3. The optical properties of the background tissue were $\mu_{a}=$ $0.1 \mathrm{~cm}^{-1}, \mu_{s}=100 \mathrm{~cm}^{-1}$, and $g=0.9$. The distance between the center of the tumor and the upper tissue surface $d_{t}$ was $1.5 \mathrm{~cm}$. Therefore the transport mean free path was

$$
L_{t}{ }^{\prime}=1 /\left[\mu_{a}+\mu_{s}(1-g)\right]=0.099 \mathrm{~cm}
$$

and the penetration depth was

$$
\delta=\left\{3 \mu_{a}\left[\mu_{a}+\mu_{s}(1-g)\right]\right\}^{1 / 2}=0.57 \mathrm{~cm} .
$$

The estimated optimal radius $r_{o}$ was $1.39 \mathrm{~cm}$, which was in good agreement with the product curve in Fig. 3.

Although the size of the tumor would affect the absolute amount of laser energy deposited into the tumor, it does not affect the choice of the optimal beam size, where the tumor is assumed to be relatively spherical and optically homogeneous and to be centered below one transport mean free path. One transport mean free path in tissues is of the order of $1 \mathrm{~mm}$ in the visible and near-IR wavelength region.

The optimal laser beam radius, given in Eq. (7) and verified by our Monte Carlo simulations (Fig. 3), is very useful for laser therapy of deep tumors. By limiting the size of the beam, the unnecessary exposure and heating of the skin surface can be minimized. By expanding the beam, one can avoid the damage caused by the peak power deposition ("hot spot") near the tissue surface. When the beam size is optimized, the power of the incident laser beam is efficiently coupled into the tumor and the power absorption by the tumor is nearly maximum. In clin- ical applications, the tumor location may be first obtained with an imaging modality such as ultrasonography, radiography, x-ray computerized tomography, magnetic resonance imaging, or optical tomography. The optimal beam size may then be estimated with Eq. (7).

Figure 5 demonstrates the effect of multiple-beam delivery. When a narrow beam was used, the power deposition under the beam was much stronger than that in the tumor. Therefore it would be easy to damage the tissue directly under the beam. When the radius of the beam broadened to 0.5 and $1 \mathrm{~cm}$, the power deposition under the beam was reduced significantly.

In Fig. 5(c) the power deposition in the tumor was comparable with that under the beam. In all cases, multiple-beam delivery, especially four-beam delivery, had clear advantages over single-beam delivery. Compared with single-beam delivery, multiple-beam delivery could yield a more homogeneous power deposition in the tumor and a higher power delivered to the tumor without causing damage to the surrounding normal tissue.

In the simulations presented in this paper, the absorption coefficient of the buried tumor was assumed to be enhanced with dyes and reached 10 times that of the background normal tissue. With the use of near-IR lasers and matched dyes, this contrast may be further increased as in the case of 805-nm laser and indocyanine green ${ }^{13-15}$ for photothermal interactions. The fact that the maximum power absorption by background tissue can be reduced with four beams to the level comparable with that absorbed by the tumor shows the promise for better therapeutic outcomes by optimizing the configuration of light delivery. Further optimization can be achieved by increasing the concentration of the dyes in tumors or increasing the number of beams. Surface cooling with liquid or gas can drastically reduce energy accumulation on the surface tissue. Scanning the laser beams around the tumor during the treatment can also reduce surface heating if the laser power can always be coupled into the tumor efficiently while the beam is scanned. The scanning approach would spread the energy absorption under the tissue surface while the tumor accumulates energy in the center of the background normal tissue.

Furthermore, when the total tumor direct killing is not the overriding objective, such as in the case of photothermal immunotherapy, ${ }^{16}$ the source power can be reduced to an even more tolerable level.

The rapid attenuation of light in turbid media can significantly reduce the energy reaching the target tumors. However, the optimal beam size, the enhanced tumor absorption, and a multiple-beam delivery system can still allow the desired selectivity for target tissue as demonstrated by this study.

Although power has been used for this study, one may change the power to energy by replacing watts with joules, and the conclusions would apply to pulse laser beams as well. Note that this study investigates only the initial optical component of laser- 
tissue interactions. Once the optical power deposition in tissues is simulated, the power deposition may be used as the source term for further investigation into photothermal, photomechanical, or photochemical effects of interest.

\section{Summary}

Monte Carlo simulations based on the deltascattering technique proved to be a flexible and powerful tool in studying light transport in heterogeneous biological tissues. From studies of power deposition in buried tumors, one concludes that an optimal radius of the laser beam existed to achieve both high-power delivery efficiency and high-power absorption by the tumor. An estimate of the optimal radius was given analytically. Multiple-beam delivery was able to yield more homogeneous and greater power absorption by the tumor than single-beam delivery without damage to the normal tissues. These results provide guidelines for various laser therapeutics including photothermal therapies, photomechanical therapies, and noninvasive photosensitizerassisted laser therapies of deep tumors.

This project was sponsored in part by the National Institutes of Health grant R29 CA68562 and The Whitaker Foundation.

\section{References and Notes}

1. M. Keijzer, J. W. Pickering, and M. J. C. van Gemert, "Laser beam diameter for port wine stain treatment," Lasers Surg. Med. 11, 601-605 (1991).

2. Q. Chen, B. C. Wilson, M. O. Dereski, M. S. Patterson, M. Chopp, and F. W. Hetzel, "Effects of light beam size on fluence distribution and depth of necrosis in superficially applied photodynamic therapy of normal rat brain," Photochem. Photobiol. 56, 379-384 (1992).

3. B. C. Wilson and G. A. Adam, "Monte Carlo model for the absorption and flux distributions of light in tissue," Med. Phys. 10, 824-830 (1983).

4. S. T. Flock, B. C. Wilson, D. R. Wyman, and M. S. Patterson, "Monte-Carlo modeling of light-propagation in highly scattering tissues I: Model predictions and comparison with diffusion theory," IEEE Trans. Biomed. Eng. 36, 1162-1168 (1989).
5. S. A. Prahl, M. Keijzer, S. L. Jacques, and A. J. Welch, "A Monte Carlo model of light propagation in tissue," in Dosimetry of Laser Radiation in Medicine and Biology, G. J. Muller and D. H. Sliney, eds., Proc. SPIE IS5, 102-111 (1989).

6. S. L. Jacques and L.-H. Wang, "Monte Carlo modeling of light transport in tissues," in Optical Thermal Response of Laser Irradiated Tissue, A. J. Welch and M. J. C. van Gemert, eds. (Plenum, New York, 1995), pp. 73-100.

7. L.-H. Wang, S. L. Jacques, and L.-Q. Zheng, "MCML-Monte Carlo modeling of photon transport in multilayered tissues," Comput. Methods Prog. Biomed. 47, 131-146 (1995). The MCML/CONV software package may be downloaded from URL: http://biomed.tamu.edu/ lw.

8. L.-H. Wang, S. L. Jacques, and L.-Q. Zheng, "CONVConvolution for responses to a finite diameter photon beam incident on multilayered tissues," to be published in Comput. Methods Prog. Biomed. (1997).

9. I. Lux and L. Koblinger, Monte Carlo Particle Transport Methods: Neutron and Photon Calculations (CRC Press, Boca Raton, Fla., 1991).

10. T. J. Farrell, M. S. Patterson, and B. C. Wilson, "A diffusion theory model of spatially resolved, steady-state diffuse reflectance for the non-invasive determination of tissue optical properties in vivo," Med. Phys. 19, 879-888 (1992).

11. L.-H. Wang and S. L. Jacques, "Use of a laser beam with an oblique angle of incidence to measure the reduced scattering coefficient of a turbid medium," Appl. Opt. 34, 2362-2366 (1995).

12. L.-H. Wang and S. L. Jacques, "Analysis of diffusion theory and similarity relations," in Photon Migration and Imaging in Random Media and Tissues, B. Chance and R. R. Alfano, eds., Proc. SPIE 1888, 107-116 (1993).

13. W. R. Chen, R. L. Adams, S. Heaton, D. T. Dickey, K. E. Bartels, and R. E. Nordquist, "Chromophore-enhanced lasertumor tissue photothermal interaction using an 808-nm diode laser," Cancer Lett. 88, 15-19 (1995).

14. W. R. Chen, R. L. Adams, K. E. Bartels, and R. E. Nordquist, "Chromophore-enhanced in vivo tumor cell destruction using an 808-nm diode laser," Cancer Lett. 94, 125-131 (1996).

15. W. R. Chen, R. L. Adams, A. K. Higgins, K. E. Bartels, and R. E. Nordquist, "Photothermal effects on murine mammary tumors using indocyanine green and an 808-nm diode laser: an in vivo efficacy study," Cancer Lett. 98, 169-173 (1995).

16. W. R. Chen, R. L. Adams, R. Carubelli, and R. E. Nordquist, "Laser-photosensitizer assisted immunotherapy: a novel modality for cancer treatment," Cancer Lett. 115, 25-30 (1997). 